\title{
ГИПЕРТЕНЗИВНЫЕ НАРУШЕНИЯ У БЕРЕМЕННЫХ ЖЕНЩИН С УЧЁТОМ ИЗМЕНЕНИЯ КЛИМАТА
}

\author{
Ф.З. ОАИМОВА, М.Ф. ДОДХОЕВА
}

Кафедра акушерства и гинекологии № 1, Таджикский государственный медицинский университет им. Абуали ибни Сино, Душанбе, Республика Таджикистан

На основании анализа мировой литературы выяснилось, что изменение климата в сторону потепления ухудшает течение беременности и перинатальные исходы. Однако результаты многочисленных исследований, проведённых в разных частях мира по изучению корреляции между температурой окружающей среды, относительной влажностью воздуха и интенсивностью солнечного света, а также частотой гипертензивных расстройств во время беременности, противоречивы. Несмотря на расхождения в результатах, в течение холодных месяцев наблюдается тенденция к более высокой заболеваемости преэклампсией. Объяснением в поддержку этой теории было бы то, что более низкие температуры влияют на заболеваемость преэклампсией и эклампсией, усиливая вазоспазм, свойственный заболеванию. Признание точной связи с разными погодными условиями может помочь понять, какие факторы могут участвовать в запуске этих процессов. Эти исследования особенно актуальны в условиях Таджикистана, где к массе экстрагенитальных заболеваний, имеющихся у беременных женщин региона, присоединяется ещё и фактор потепления климата.

Ключевые слова: гипертензивные нарушения, беременность, климат, сезонность.

Для цитирования: Олимова Ф3, Додхоева МФ. Гипертензивные нарушения у беременных женщин с учётом изменения климата. Вестник АвиценHы. 2018;20(4):467-72. Available from: http://dx.doi.org/10.25005/2074-0581-2018-20-4-467-472

\section{HYPERTENSIVE DISORDERS IN PREGNANT WOMEN SUBJECT TO THE CLIMATE CHANGE}

\author{
F.Z. OLIMOVA, M.F. DODKHOEVA
}

Department of Obstetrics and Gynecology № 1, Avicenna Tajik State Medical University, Dushanbe, Republic of Tajikistan

Based on the analysis of the world literature, it turned out that climate change in the direction of warming worsens pregnancy and perinatal outcomes. However, the results of numerous studies conducted in different parts of the world to study the correlation between ambient temperature, relative humidity and intensity of sunlight, as well as the frequency of hypertensive disorders during pregnancy, are contradictory. Despite differences in results, a tendency to a higher incidence of preeclampsia is observed during the cold months. An explanation in support of this theory would be that more low temperatures affect the incidence of preeclampsia and eclampsia, increasing the vasospasm inherent in the disease. Recognizing the exact relationship with different weather conditions can help you understand what factors may be involved in the launch of these processes. These studies are particularly relevant in the conditions of Tajikistan, where the mass of extragenital diseases present in pregnant women in the region is also joined by the climate warming factor.

Keywords: Hypertensive disorders, pregnancy, climate, seasonality.

For citation: Olimova FZ, Dodkhoeva MF. Gipertenzivnye narusheniya u beremennykh zhenshchin s uchyotom izmeneniya klimata [Hypertensive disorders in pregnant women subject to the climate change]. Vestnik Avitsenny [Avicenna Bulletin]. 2018;20(4):467-72. Available from: http://dx.doi.org/10.25005/2074 0581-2018-20-4-467-472

B последние годы, в результате человеческой деятельности, сжигания ископаемых видов топлива, в нижних слоях атмосферы скопились углекислый газ и другие парниковые газы в количествах, достаточных для удержания излишнего тепла и воздействия на глобальный климат. За последние 130 лет температура в мире возросла примерно на $0,85^{\circ} \mathrm{C}$, а за последние 25 лет темпы глобального потепления ускорились, превысив $0,18^{\circ} \mathrm{C}$ за десятилетие (Intergovernmental Panel on Climate Change, 2014). Как ожидается, изменение климата вызовет порядка 250000 смертей дополнительно в год в период с 2030 по 2050 (WHO, 2014). Во время сильной жары повышаются уровни пыльцы растений и других аэроаллергенов. Они провоцируют бронхиальную астму, от которой страдает около 300 миллионов человек. Ожидается, что продолжающееся повышение температуры усилит это бремя (ВО3, 2016).

Беременные женщины относятся к числу субпопуляций, наиболее уязвимых к воздействию экстремальных температурных условий. Увеличение жировых отложений и уменьшение площади поверхности тела по соотношению с массой тела, из- за увеличения веса во время беременности, могут уменьшить способность женщины терять тепло в окружающей среде (Р. Dadvand, 2017). Имеет место также увеличение выработки внутреннего тепла из-за роста плода и обмена веществ. Эти изменения ограничивают способность беременных женщин смягчать тепловое напряжение [1].

S. Ha et al (2017) обследовали 680 рожениц с одноплодной беременностью, страдающих от сердечно-сосудистых заболеваний, в 12 городах США (2002-2008 гг.). Оценивалась средняя суточная температура воздуха за неделю до родов и в день родов. В тёплое время года риски были более выражены в дни, близкие к родам, а чернокожие женщины оказались более восприимчивыми к такому повышению температуры [2].

Приблизительно 17-19\% случаев мёртворождения потенциально могут быть вследствие хронических рисков в течение всей беременности и локальных экстремальных температур. Имеются данные, что увеличение температуры воздуха на $1^{\circ} \mathrm{C}$ в течение недели, предшествовавшего родам, было связано с увеличением риска мёртворождения на 6\% (3-9\%), в течение тёпло- 
го сезона (май-сентябрь). Это явление приводит приблизительно к 4 (2-6) дополнительным мёртворождениям на 10000 родов при каждом увеличении на $1^{\circ} \mathrm{C}[3]$.

Изменение климата в сторону потепления влияет не только на здоровье матерей, но и ухудшает неонатальные исходы. Недавние исследования показывают, что повышение температуры в тёплые сезоны могут увеличить риск преждевременной отслойки плаценты у женщин, чья беременность доношенная или близка к сроку. Авторы рекомендуют учитывать профилактические меры, такие как кондиционирование воздуха и гидратацию в жаркую погоду [4, 5].

Kuehn L. et McCormick S. (2017), систематически рассматривая статьи из обзоров PubMed и Cochrane, включили работы, в которых описываются воздействия, связанные с изменением климата, и неблагоприятные последствия на здоровье беременных женщин. Имеются данные о том, что экстремальная температура отрицательно влияет на исход беременности, включая изменения в продолжительности беременности, веса при рождении, антенатальную гибель плода и неонатальный стресс. Исследования, включённые в этот обзор, показывают, что существует не только необходимость в дальнейших исследованиях того, как изменение климата, в частности, высокая температура, может влиять на состояние здоровья матерей и неонатальные исходы, но и изучить эти единые стандарты для оценки воздействия тепла на здоровье плода [6-9].

Изучая влияние климатических изменений на гипертензивные нарушения у беременных, авторы пришли к выводу, что данное осложнение гестации и его последствия, такие как преждевременные роды, низкая масса тела при рождении, распространены в холодные и влажные сезоны. Интенсивность солнечного света в период оплодотворения была связана с более высокими показателями преэклампсии (ПЭ), эклампсии, преждевременных родов и катаракты $[10,11]$.

Влияние на заболеваемость таких экологических условий, как температура, относительная влажность воздуха и сезоны года, было признано со времён Гиппократа, когда эти факторы влияли на частоту инфекционных заболеваний. В последние годы сезонное влияние было признано также в случаях таких неинфекционных заболеваний, как сердечно-сосудистые и психические [12-14]. Аналогично, были зафиксированы наблюдения за воздействием условий окружающей среды на частоту гипертензивных нарушений у беременных, от которых в мире страдает около $10 \%$ беременных [15-19]. Хотя эти исследования остаются противоречивыми, недавний систематический обзор по этому вопросу обнаружил неоднозначные свидетельства сезонных изменений в происхождении гипертензивных расстройств при беременности в тропических и нетропических регионах [20].

Исследование по поводу взаимосвязи сезонных изменений с преэклампсией, проведённое в Бразилии, показало самую низкую $(9,95 \%)$ частоту этой патологии в феврале и наибольшую (21,54\%) - в августе. В течение изученных лет средняя месячная температура колебалась от $24^{\circ} \mathrm{C}$ до $27^{\circ} \mathrm{C}$, при этом не было значительных изменений относительной влажности воздуха (69-87\%). Как упоминалось выше, результаты многочисленных исследований, проведённых по корреляции между температурой окружающей среды, относительной влажностью воздуха и интенсивностью солнечного света, с одной стороны, а также частотой гипертензивных расстройств во время беременности, с другой, противоречивы. В исследовании, проведённом в течение 36-месячного периода у 11958 новорождённых в штате Миссисипи, США, которое было посвящено выявлению гипер- тензивных расстройств во время беременности у их матерей и их распределению в течение месяцев года, в соответствии со средней температурой и относительной влажностью воздуха, корреляции между частотой гипертензивных расстройств и сезонностью не обнаружено [12]. Однако в другом исследовании, проведённом в Киншасе, Конго, с 17592 женщинами, отмечалась более высокая заболеваемость преэклампсией в сухие месяцы года (13\% против 6\%) [21].

В Индии было проведено исследование с целью определить наличие сходной корреляции между гипертензивными расстройствами беременности и муссонными месяцами (июнь, июль и август), когда влажность достигает $85 \%$, и другими месяцами года, когда влажность составляет 70\%. Из 29562 случаев, зарегистрированных в этом исследовании, у 1238 пациенток (4,18\%) развилась ПЭ, и из них у 34 (0,11\%) - перешла в эклампсию. Не было обнаружено корреляции между частотой ПЭ и двумя исследованными периодами, тогда как между эпизодами эклампсии и сухим сезоном была обнаружена значительная корреляция по сравнению с сезоном дождей $(0,2 \%$ против $0,08 \%, p=0,01)$ [16].

Ретроспективное исследование, проведённое A. Immink et al (2008), проанализировало более 11000 родов в больнице Тигерберг, в Южной Африке, и обнаружило, что случаи ПЭ наиболее часто $(13,6 \%)$ встречались в зимний период $(13,6 \%)$ [22]. W.H. Tam et al (2008), также отмечали сезонные тенденции в распространении ПЭ у первородящих в Гонконге в период с 1995 по 2002 г.г.: пациентки, поступившие в июне, имели наивысший риск развития ПЭ по сравнению с женщинами, которые наблюдались в октябре. Авторы пришли к выводу, что необходимы дальнейшие исследования для изучения роли температуры и влажности окружающей среды в развитии ПЭ [15].

J.K. Phillips et al (2004) изучали, были ли время родов или время зачатия более тесно связаны с развитием ПЭ. Исследованию подверглись 7904 первородящих, беременность которых в 142 случаях была осложнена преэклампсией. Авторы обнаружили сезонные корреляции ПЭ, которые, по-видимому, были более тесно связаны со временем зачатия, чем со временем родов. Зачатие в течение летних месяцев по сравнению с весной было связано с увеличением вероятности ПЭ на 70\% [23]. В подобной работе о сезонной корреляции ПЭ в Техасе выявлено, что сезонные колебания были минимальными с наименьшей распространённостью осенью (3,89\%) и пиком зимой, в январе (4,4\%) [24].

В Китае наибольшая распространённость гестационной гипертензии выявлена в зимне-весенний период [25]. Результаты исследования учёных в восточном Судане показывают высокую частоту ПЭ в зимний период [26], что совпадает с данными S. Shahgheibi et al (2016) [27]. В аналогичном исследовании, проведённом в Японии, также установлено, что пик гипертензивных нарушений был отмечен в зимние месяцы (январь и февраль), а самая низкая частота $(4,1 \%)$ отмечалась в июле и августе, когда влажность воздуха была наивысшая. Хотя месячный показатель распространённости гипертензивных нарушений в зависимости от месяца при зачатии значительно колебался, показатель частоты был самым высоким (4,9\%) для женщин с зачатием в марте и апреле и самым низким (2,9\%) для женщин с зачатием в ноябре и декабре [28]. Исходя из времени зачатия, в Иране наибольшая распространённость Пэ была обнаружена летом, особенно в сентябре $(11,06875 \%)$ и августе $(10,3053 \%)$, а самая низкая зимой и ранней весной, особенно в январе $(5,7552 \%)$ и апреле (5,7252 \%). Учёные считают, что сезонные (месячные) изменения погоды (влажность и температура) оказывают значительное влияние на распространённость ПЭ $[29,30]$. 
Д.А. Каюмова (2011), проанализировав истории родов 6595 женщин репродуктивного возраста от 15 до 49 лет, установила, что беременные и роженицы наиболее уязвимы в жаркий период года, чем зимой, так как показатели взаимозависимости сопутствующих заболеваний летом выше, чем зимой - особенно коэффициент парной корреляции между дефицитом массы тела и анемией $(r=0,71)$ значительно выше летом, чем зимой $(r=0,05)$. Исследователем было установлено, что женщины, которые впервые рожают в жаркий период, наиболее уязвимы к развитию гипертензивных нарушений, чем зимой. Наряду с этим, было установлено, что летом гипертензивные нарушения наиболее часто наблюдаются в первой половине суток, а зимой - во второй [5].

Признание точной связи с разными погодными условиями может помочь нам понять, какие факторы участвуют в запуске этих процессов. Несмотря на расхождения в результатах, сообщённых в разных частях мира относительно возможного влияния окружающей среды на заболеваемость гипертензивными расстройствами во время беременности, в течение более холодных месяцев, как видно, наблюдается тенденция к более высокой заболеваемости ПЭ. Объяснением в поддержку этой теории было бы то, что более низкие температуры влияют на заболеваемость ПЭ и эклампсией, усиливая вазоспазм, свойственный этой патологии [12], по сравнению с жаркими сезонами, способствующими вазодилатации и снижению объёма циркулирующей крови [20]. Также установлено, что у детей с неонатальной энцефалопатией в раннем неонатальном периоде, который приходится на холодную часть года, имеется напряжённость системы гемостаза с компенсированным внутрисосудистым свёртыванием крови [31].

Более тёплая температура сухих сезонов вызывает значительную потерю жидкости, что может играть защитную роль в возникновении эклампсии [16]. Согласно сообщениям ряда авторов, увеличение температуры воздуха на $10^{\circ} \mathrm{C}$ снижает как систолическое, так и диастолическое АД в среднем на 2,5 мм рт. ст. [32]. Изучая сезонное различие АД у беременных, исследователи пришли к выводу, что уровни систолического и диастолического АД на 1,0-1,7 мм рт. ст. выше в январе, на 0,6 мм рт. ст. в сентябре и октябре, и на 0,8 мм рт. ст. в ноябре и декабре по сравнению с августом [33].

Однако результаты исследования, проведённого в Греции с целью установления сезонной взаимосвязи ПЭ, показали, что в большинстве случаев тяжёлая ПЭ была зарегистрирована летом, хотя статистическая разница не была значительной [34]. Другие авторы также сообщили, что с увеличением температуры воздуха и влажности возрастает и заболеваемость ПЭ [35]. Возможное объяснение этого пика в летнее время может быть следствием продуцирования вазоактивных веществ и удерживания жидкости в жаркие месяцы в качестве защитного механизма организма против обезвоживания.

Будущие эпидемиологические исследования сезонных тенденций в развитии ПЭ, по-видимому, должны быть направлены на изучение влияния таких факторов, как время, проведённое беременной на открытом воздухе и связанное с этим, в частности, содержание витамина D в материнской сыворотке, низкий уровень которого был связан с тяжёлой ПЭ [24]. О возможной роли витамина D в развитии ПЭ, включая иммунную дисфункцию и плацентарную имплантацию, сообщается в ряде работ [36-38].

Таким образом, на основании анализа мировой литературы выяснилось, что изменение климата в сторону потепления ухудшает течение беременности и перинатальные исходы. Результаты других многочисленных исследований в разных частях мира, проведённых по выявлению корреляции между температурой окружающей среды, относительной влажностью воздуха и интенсивностью солнечного света, а также частотой гипертензивных расстройств во время беременности, противоречивы, что требует проведения более глубоких патогенетических исследований.

\section{ЛИТЕРАТУРА}

1. Dadvand P. Congenital anomalies: an under-evaluated risk of climate change. Occup Environ Med. 2017;74:313-4. Available from: http://dx.doi. org/10.1136/oemed-2016-104193.

2. Ha S, Nguyen K, Liu D, Männistö T, Nobles C, Sherman S, et al. Ambient temperature and risk of cardiovascular events at labor and delivery: A casecrossover study. J Environ Res. 2017;159:622-8. Available from: https:// dx.doi.org/10.1016/j.envres.2017.09.010.

3. Ha S, Liu D, Zhu Y, Soo Kim S, Sherman S, Grantz KL, et al. Ambient temperature and stillbirth: A multi-center retrospective cohort study. $J$ Environ Health Perspect. 2017;125(6):945. Available from: https://dx.doi. org/10.1289/EHP945.

4. He S, Kosatsky T, Smargiassi A, Bilodeau-Bertrandab M, Augerabce N. Heat and pregnancy-related emergencies: Risk of placental abruption during hot weather. Environment International. 2018;111:295-300. Available from:https://doi.org/10.1016/j.envint.2017.11.004.

5. Додхоева МФ, Рафиев МК, Каюмова ДА, Сабурова ХШ. Некоторые аспекты здоровья населения Таджикистана в условиях потепления климата. Доклады Академии наук Республики Таджикистан. 2014;57(7):606-11.

6. Kuehn L, McCormick S. Heat exposure and maternal health in the face of climate change. Int J Environ Res Public Health. 2017;14(8):853. Available from: https://dx.doi.org/doi:10.1111/risa.12767.

7. Cil G, Cameron TA. Potential climate change health risks from increases in heat waves: abnormal birth outcomes and adverse maternal health

\section{REFERENCES}

1. Dadvand P. Congenital anomalies: an under-evaluated risk of climate change. Occup Environ Med. 2017;74:313-4. Available from: http://dx.doi. org/10.1136/oemed-2016-104193.

2. Ha S, Nguyen K, Liu D, Männistö T, Nobles C, Sherman S, et al. Ambient temperature and risk of cardiovascular events at labor and delivery: A casecrossover study. J Environ Res. 2017;159:622-8. Available from: https:// dx.doi.org/10.1016/j.envres.2017.09.010.

3. Ha S, Liu D, Zhu Y, Soo Kim S, Sherman S, Grantz KL, et al. Ambient temperature and stillbirth: A multi-center retrospective cohort study. Environ Health Perspect. 2017;125(6):945. Available from: https://dx.doi. org/10.1289/EHP945.

4. He S, Kosatsky T, Smargiassi A, Bilodeau-Bertrandab M, Augerabce N. Heat and pregnancy-related emergencies: Risk of placental abruption during hot weather. Environment International. 2018;111:295-300. Available from:https://doi.org/10.1016/j.envint.2017.11.004.

5. Dodkhoeva MF, Rafiev MK, Kayumova DA, Saburova KS. Nekotorye aspekty zdorov'ya naseleniya Tadzhikistana $v$ usloviyakh potepleniya klimata [Some aspects of health of the population of Tajikistan in conditions of warming of the climate]. Doklady Akademii nauk Respubliki Tadzhikistan. 2014;57(7):606-11.

6. Kuehn L, McCormick S. Heat exposure and maternal health in the face of climate change. Int J Environ Res Public Health. 2017;14(8):853. Available from: https://dx.doi.org/doi:10.1111/risa.12767.

7. Cil G, Cameron TA. Potential climate change health risks from increases in heat waves: abnormal birth outcomes and adverse maternal health 
conditions. J Risk Anal. 2017;37(11):2066-79. Available from: https://dx.doi. org/doi:10.1111/risa.12767.

8. Guo $T$, Wang $Y$, Zhang $H$, Zhang $Y$, Zhao J, Wang $Y$. The association between ambient temperature and the risk of preterm birth in China. Sci Total Environ. 2018;613-614:439-46. Available from: https://dx.doi.org/doi:10.1016/j. scitotenv.2017.09.104.

9. Carolan-Olah M, Frankowska D. High environmental temperature and preterm birth: a review of the evidence. J Midwifery. 2014;30(1):50-9. Available from: https://dx.doi.org/doi:10.1016/j.midw.2013.01.011.

10. Poursafa P, Keikha M, Kelishadi R. Poursafa P. Systematic review on adverse birth outcomes of climate change. J Res Med Sci. 2015;20(4):397-402.

11. Auger N, Siemiatycki J, Bilodeau-Bertrand M, Healy-Profitós J, Kosatsky T. Ambient temperature and risk of preeclampsia: biased association? Paediatric and Perinatal Epidemiology. 2017;31(4):267-71. Available from: https://doi.org/10.1111/ppe.12362.

12. Melo B, Amorim M, Katz L, Coutinho I, Figueiroa JN. Hypertension, pregnancy and weather: is seasonality involved? Rev Assoc Med Bras. 2014;60(2):10510. Available from: http://dx.doi.org/10.1590/1806-9282.60.02.006.

13. Коваленко МС, Ефремова МГ, Окорочкова ЮВ. Особенности течения беременности и родоразрешения первородящих критических возрастных групп. Наука молодых. 2014;1:94-9.

14. Петрова ЕИ. Состояние и перспективы развития лечебно-профилактической помощи беременным и новорождённым в Рязанской области. Наука молодых. 2013;1:46-50.

15. Tam WH, Sahota DS, Lau TK, Li CY, Fung TY. Seasonal variation in pre-eclamptic rate and its association with the ambient temperature and humidity in early pregnancy. Gynecol Obstet Invest. 2008;66(1):22-6. Available from: https:// doi.org/10.1159/000114252.

16. Subramaniam V. Seasonal variation in the incidence of preeclampsia and eclampsia in tropical climatic conditions. BMC Women's Health. 2007;7:18. Available from: https://doi.org/10.1186/1472-6874-7-18.

17. Roberts JM, August AP, Bakris G, Barton JR, Bernstein IM, Druzin M, et al. Hypertension, pregnancy-induced - practice guideline. Washington, USA: American College of Obstetricians and Gynecologists; 2013. 89 p.

18. Радзинский ВЕ, Фукс ВЕ. (ред.) Акушерство. Москва, РФ: ГЭОТАР-Медиа; 2016. 608 c.

19. Додхоева МФ, Олимова ФЗ. Современные теории этиопатогенеза и профилактики преэклампсии. Вестник Авиценны. 2017;19(4):537-43. Available from: http://dx.doi.org/10.25005/2074-0581-2017-19-4-537-543.

20. TePoel MR, Saftlas AF, Wallis AB. Association of seasonality with hypertension in pregnancy: a systematic review. J Reprod Immunol. 2011;89(2):140-52. Available from:https://doi.org/10.1016/j.jri.2011.01.020.

21. Elongi JP, Tandu B, Spitz B, Verdonck F. Infuence of the seasonal variation on the prevalence of preeclampsia in Kinshasa. Gynecol Obstet Fertil. 2011;39(3):132-5. Available from: http://dx.doi.org/doi: 10.1016/j. gyobfe.2010.12.010.

22. Immink A, Scherjon S, Wolterbeek R, Wilhelm Steyn D. Seasonal influence on the admittance of pre-eclampsia patients in Tygerberg Hospital. J Acta Obstet Gynecol Scand. 2008;87(1):36-42. Available from: https://doi. org/10.1080/00016340701743066.

23. Phillips JK, Bernstein IM, Mongeon JA, Badger GJ. Seasonal variation in preeclampsia based on timing of conception. Obstet Gynecol. 2004;104(5):101520. Available from:https://10.1097/01.AOG.0000143306.88438.cf.

24. Wellington K, Mulla ZD. Seasonal trend in the occurrence of preeclampsia and eclampsia in Texas. American Journal of Hypertension. 2012;25(2):115-9. Available from: https://doi.org/10.1038/ajh.2011.173.

25. Li X, Tan H, Huang X, Zhou S, Hu S, Wang X, et al. Similarities and differences between the risk factors for gestational hypertension and preeclampsia: A population based cohort study in south China. An International Journal of Women's Cardiovascular Health. 2016;6(1):66-71. Available from: https:// doi.org/10.1016/j.preghy.2015.11.004.

26. Ali AA, Adam, GK, Abdallah TM. Seasonal variation and hypertensive disorders of pregnancy in eastern Sudan. Journal of Obstetrics and Gynaecology. 2015; 35(2):153-4. Available from: https://doi.org/10.3109/01443615.2014.94881 5.

27. Shahgheibi S, Rezaie M, Kamangar TM, Zarea S, Youse SR. The effect of season on the prevalence of preeclampsia. J Clin Gynecol Obstet. 2016;5(3):81-4. Available from: https://doi.org/10.14740/jcgo408w. conditions. J Risk Anal. 2017;37(11):2066-79. Available from: https://dx.doi. org/doi:10.1111/risa.12767.

8. Guo $\mathrm{T}$, Wang $\mathrm{Y}$, Zhang $\mathrm{H}$, Zhang $\mathrm{Y}$, Zhao J, Wang $\mathrm{Y}$. The association between ambient temperature and the risk of preterm birth in China. Sci Total Environ. 2018;613-614:439-46. Available from: https://dx.doi.org/doi:10.1016/j. scitotenv.2017.09.104.

9. Carolan-Olah M, Frankowska D. High environmental temperature and preterm birth: a review of the evidence. J Midwifery. 2014;30(1):50-9. Available from: https://dx.doi.org/doi:10.1016/j.midw.2013.01.011.

10. Poursafa P, Keikha M, Kelishadi R. Poursafa P. Systematic review on adverse birth outcomes of climate change. J Res Med Sci. 2015;20(4):397-402.

11. Auger N, Siemiatycki J, Bilodeau-Bertrand M, Healy-Profitós J, Kosatsky T. Ambient temperature and risk of preeclampsia: biased association? Paediatric and Perinatal Epidemiology. 2017;31(4):267-71. Available from: https://doi.org/10.1111/ppe.12362.

12. Melo B, Amorim M, Katz L, Coutinho I, Figueiroa JN. Hypertension, pregnancy and weather: is seasonality involved? Rev Assoc Med Bras. 2014;60(2):10510. Available from: http://dx.doi.org/10.1590/1806-9282.60.02.006.

13. Kovalenko MS, Efremova MG, Okorochkova YuV. Osobennosti techeniya beremennosti i rodorazresheniya pervorodyashchikh kriticheskikh vozrastnykh grupp [The peculiarities of gestational process and delivery in case of critical age group primipara]. Nauka molodykh. 2014;1:94-9.

14. Petrova El. Sostoyanie i perspektivy razvitiya lechebno-profilakticheskoy pomoshchi beremennym i novorozhdyonnym v Ryazanskoy oblasti [State and prospects of development of the treatment-and-prophylactic aid to pregnant women and to newborns in the Ryazan region]. Nauka molodykh. 2013;1:46-50.

15. Tam WH, Sahota DS, Lau TK, Li CY, Fung TY. Seasonal variation in pre-eclamptic rate and its association with the ambient temperature and humidity in early pregnancy. Gynecol Obstet Invest. 2008;66(1):22-6. Available from: https:// doi.org/10.1159/000114252.

16. Subramaniam V. Seasonal variation in the incidence of preeclampsia and eclampsia in tropical climatic conditions. BMC Women's Health. 2007;7:18. Available from: https://doi.org/10.1186/1472-6874-7-18.

17. Roberts JM, August AP, Bakris G, Barton JR, Bernstein IM, Druzin M, et al. Hypertension, pregnancy-induced - practice guideline. Washington, USA: American College of Obstetricians and Gynecologists; 2013. 89 p.

18. Radzinskiy VE, Fuks VE. (red.) Akusherstvo [Obstetrics]. Moscow, RF: GEOTAR-Media; 2016. 608 p.

19. Dodkhoeva MF, Olimova FZ. Sovremennye teorii etiopatogeneza i profilaktiki preeklampsii [Modern theories of etiopathogenesis and prevention of preeclampsia]. Vestnik Avitsenny [Avicenna Bulletin]. 2017;19(4):537-43. Available from: http://dx.doi.org/10.25005/2074-0581-2017-19-4-537-543.

20. TePoel MR, Saftlas AF, Wallis AB. Association of seasonality with hypertension in pregnancy: a systematic review. J Reprod Immunol. 2011;89(2):140-52. Available from:https://doi.org/10.1016/j.jri.2011.01.020.

21. Elongi JP, Tandu B, Spitz B, Verdonck F. Infuence of the seasonal variation on the prevalence of preeclampsia in Kinshasa. Gynecol Obstet Fertil. 2011;39(3):132-5. Available from: http://dx.doi.org/doi: 10.1016/j. gyobfe.2010.12.010.

22. Immink A, Scherjon S, Wolterbeek R, Wilhelm Steyn D. Seasonal influence on the admittance of pre-eclampsia patients in Tygerberg Hospital. J Acta Obstet Gynecol Scand. 2008;87(1):36-42. Available from: https://doi. org/10.1080/00016340701743066.

23. Phillips JK, Bernstein IM, Mongeon JA, Badger GJ. Seasonal variation in preeclampsia based on timing of conception. Obstet Gynecol. 2004;104(5):101520. Available from:https://10.1097/01.AOG.0000143306.88438.cf.

24. Wellington K, Mulla ZD. Seasonal trend in the occurrence of preeclampsia and eclampsia in Texas. American Journal of Hypertension. 2012;25(2):115-9. Available from: https://doi.org/10.1038/ajh.2011.173.

25. Li X, Tan H, Huang X, Zhou S, Hu S, Wang X, et al. Similarities and differences between the risk factors for gestational hypertension and preeclampsia: $A$ population based cohort study in south China. An International Journal of Women's Cardiovascular Health. 2016;6(1):66-71. Available from: https:// doi.org/10.1016/j.preghy.2015.11.004.

26. Ali AA, Adam, GK, Abdallah TM. Seasonal variation and hypertensive disorders of pregnancy in eastern Sudan. Journal of Obstetrics and Gynaecology. 2015; 35(2):153-4. Available from: https://doi.org/10.3109/01443615.2014.94881 5.

27. Shahgheibi S, Rezaie M, Kamangar TM, Zarea S, Youse SR. The effect of season on the prevalence of preeclampsia. J Clin Gynecol Obstet. 2016;5(3):81-4. Available from: https://doi.org/10.14740/jcgo408w. 
28. Morikawa M, Yamada T, Yamada T, Cho K, Sato S, Minakami H. Seasonal variation in the prevalence of pregnancy-induced hypertension in Japanese women. J Obstetrics and Gynaecology Research. 2014;40(4):926-31. Available from: https://doi.org/10.1111/jog.12304.

29. Nasiri R, Shadmehri A, Ghiassi P, Yazdi M, Baf M. Association of meteorological factors and seasonality with preeclampsia: a 5-year study in northeast of Iran. Journal Clinical and Experimental Hypertension. 2014;36(8):586-9. Available from:https://doi.org/10.3109/10641963.2014.881847.

30. Mohaddesi $H$, Nanbakhsh F. Seasonal variation in the incidence of preeclampsia based on the time of conception. World Applied Sciences Journal. 2013;28(12):2242-8

31. Хотамова МН, Додхоев ДС, Мамаджанова ГС, Аминов ХД. Основные показатели гемостаза в зимний период у новорождённых с неонатальной энцефалопатией. Вестник Авиценны. 2017;19(2):231-5. Available from: http://dx.doi.org/10.25005/2074-0581-2017-19-2-230-235.

32. Metoki H, Ohkubo T, Watanabe $\mathrm{Y}$, Misatoa N, Sato, Yuriea K, et al. Seasonal trends of blood pressure during pregnancy in Japan: The babies and their parents' longitudinal observation in Suzuki Memorial Hospital in Intrauterine Period study. J Hypertens. 2008;26(12):2406-13. Available from: https://doi. org/10.1097/HJH.0b013e32831364a7.

33. Bodnar LM, Daftary A, Markovic N, Schatzman CL, Roberts JM. Seasonal variation in gestational blood pressure. J Hypertens Preg. 2006;25:271-83. Available from: https://doi.org/10.1080/10641950600913057.

34. Daniilidis A, Chastamouratidis C, Balaouras D, Chitzios D, Loufopoulos PD, et al Seasonal variation in the incidence of severe preeclampsia in Mediterranean climatic conditions. European Journal of Inflammation. 2013;11(2):527-9. Available from: https://doi.org/10.1177/1721727X1301100222.

35. Janani F, Changaee F. Seasonal variation in the prevalence of preeclampsia. Journal of Family Medicine and Primary Care. 2017;6(4):766-9. Available from: https://doi.org/10.4103/jfmpc.jfmpc_132_17.

36. Bodnar LM, Catov JM, Simhan HN, Holick MF, Powers RW, Roberts JM Maternal vitamin D deficiency increases the risk of preeclampsia. J Clin Endocrinol Metab. 2007;92(9):3517-22. Available from: https://doi. org/10.1210/jc.2007-0718.

37. Baker AM, Haeri S, Camargo CA Jr, Espinola JA, Stuebe AM. A nested casecontrol study of midgestation vitamin $D$ deficiency and risk of severe preeclampsia. J Clin Endocrinol Metab. 2010;95(11):5105-9. Available from: https://doi.org/10.1210/jc.2010-0996.

38. Zabul P, Wozniak M, Slominski AT, Preis K, Gorska M, Korozan M, et al. A proposed molecular mechanism of high-dose vitamin D3 supplementation in prevention and treatment of preeclampsia. Int J Mol Sci. 2015;16(6):1304364. Available from: http://dx.doi.org/10.3390/ijms160613043.
28. Morikawa M, Yamada T, Yamada T, Cho K, Sato S, Minakami H. Seasonal variation in the prevalence of pregnancy-induced hypertension in Japanese women. J Obstetrics and Gynaecology Research. 2014;40(4):926-31. Available from: https://doi.org/10.1111/jog.12304.

29. Nasiri R, Shadmehri A, Ghiassi P, Yazdi M, Baf M. Association of meteorological factors and seasonality with preeclampsia: a 5-year study in northeast of Iran. Journal Clinical and Experimental Hypertension. 2014;36(8):586-9. Available from:https://doi.org/10.3109/10641963.2014.881847.

30. Mohaddesi H, Nanbakhsh F. Seasonal variation in the incidence of preeclampsia based on the time of conception. World Applied Sciences Journal. 2013;28(12):2242-8.

31. Khotamova MN, Dodkhoev DS, Mamadzhanova GS, Aminov KhD. Osnovnye pokazateli gemostaza $v$ zimniy period $u$ novorozhdyonnykh $s$ neonatal'noy entsefalopatiey [The main hemostatic profiles of newborn infant with neonatal encephalopathy in winter period]. Vestnik Avitsenny [Avicenna Bulletin]. 2017;19(2):231-5. Available from: http://dx.doi. org/10.25005/2074-0581-2017-19-2-230-235.

32. Metoki H, Ohkubo T, Watanabe $Y$, Misatoa N, Sato, Yuriea K, et al. Seasonal trends of blood pressure during pregnancy in Japan: The babies and their parents' longitudinal observation in Suzuki Memorial Hospital in Intrauterine Period study. J Hypertens. 2008;26(12):2406-13. Available from: https://doi. org/10.1097/HJH.0b013e32831364a7.

33. Bodnar LM, Daftary A, Markovic N, Schatzman CL, Roberts JM. Seasonal variation in gestational blood pressure. J Hypertens Preg. 2006;25:271-83. Available from: https://doi.org/10.1080/10641950600913057.

34. Daniilidis A, Chastamouratidis C, Balaouras D, Chitzios D, Loufopoulos PD, et al. Seasonal variation in the incidence of severe preeclampsia in Mediterranean climatic conditions. European Journal of Inflammation. 2013;11(2):527-9. Available from: https://doi.org/10.1177/1721727X1301100222.

35. Janani F, Changaee F. Seasonal variation in the prevalence of preeclampsia. Journal of Family Medicine and Primary Care. 2017;6(4):766-9. Available from: https://doi.org/10.4103/jfmpc.jfmpc_132_17.

36. Bodnar LM, Catov JM, Simhan HN, Holick MF, Powers RW, Roberts JM Maternal vitamin D deficiency increases the risk of preeclampsia. J Clin Endocrinol Metab. 2007;92(9):3517-22. Available from: https://doi. org/10.1210/jc.2007-0718.

37. Baker AM, Haeri S, Camargo CA Jr, Espinola JA, Stuebe AM. A nested casecontrol study of midgestation vitamin $D$ deficiency and risk of severe preeclampsia. J Clin Endocrinol Metab. 2010;95(11):5105-9. Available from: https://doi.org/10.1210/jc.2010-0996.

38. Zabul P, Wozniak M, Slominski AT, Preis K, Gorska M, Korozan M, et al. A proposed molecular mechanism of high-dose vitamin D3 supplementation in prevention and treatment of preeclampsia. Int J Mol Sci. 2015;16(6):1304364. Available from: http://dx.doi.org/10.3390/ijms160613043.

\section{(1) СведенИЯ ОБ Авторах}

Олимова Фарангис Зафаровна, аспирантка кафедры акушерства и гинекологии № 1, Таджикский государственный медицинский университет им. Абуали ибни Сино

Додхоева Мунаввара Файзуллоевна, академик АМН РТ, доктор медицинских наук, профессор, профессор кафедры акушерства и гинекологии № 1, Таджикский государственный медицинский университет им. Абуали ибни Сино

Информация об источнике поддержки в виде грантов, оборудования, лекарственных препаратов

Финансовой поддержки со стороны компаний-производителей лекарственных препаратов и оборудования авторы не получали.

\section{AUTHOR INFORMATION}

Olimova Farangis Zafarovna, Postgraduate Student of the Department of Obstetrics and Gynecology № 1, Avicenna Tajik State Medical University

Dodkhoeva Munavvara Fayzulloevna, Academician of the Academy of Medical Sciences of the Republic of Tajikistan, Doctor of Medical Sciences, Full Professor, Professor of the Department of Obstetrics and Gynecology № 1, Avicenna Tajik State Medical University

Конфликт интересов: отсутствует. 


\section{$\triangle$ АДРЕС ДЛЯ КОРРЕСПОНДЕНЦИИ:}

\section{Додхоева Мунаввара Файзуллоевна}

академик АМН РТ, доктор медицинских наук, профессор, профессор кафедры акушерства и гинекологии № 1 , Таджикский государственный медицинский университет им. Абуали ибни Сино

734003, Республика Таджикистан, г. Душанбе, пр. Рудаки, 139

Тел.: +992 (918) 612606

E-mail: dodkho2008@mail.ru

ВКЛАД АВТОРОВ
Разработка концепции и дизайна исследования: ДМФ
Сбор материала: ОФЗ
Анализ полученных данных: ДМФ
Подготовка текста: ДМФ, ОФЗ
Редактирование: ДМФ
Общая ответственность: ДМФ

\section{ADDRESS FOR CORRESPONDENCE:}

\section{Dodkhoeva Munavvara Fayzulloevna}

Academician of the Academy of Medical Sciences of the Republic of Tajikistan, Doctor of Medicine, Full Professor, Professor of the Department of Obstetrics and Gynecology № 1, Avicenna Tajik State Medical University

734003, Republic of Tajikistan, Dushanbe, Rudaki Ave., 139 Tel.: +992 (918) 612606

E-mail: dodkho2008@mail.ru

Submitted $\quad 08.08 .2018$

Accepted $\quad 14.11 .2018$ 